randomized to additionally receive either rosiglitazone $(n=1,117)$ or a sulfonylurea $(n=1,105)$. In this interim analysis, Home et al. compared the outcomes of all patients assigned rosiglitazone combinations with those of patients assigned metformin plus sulfonylurea (controls). Over a mean follow-up of 3.75 years, the incidence of adjudicated cardiovascular events resulting in hospitalization or death in the rosiglitazone group was not significantly different from that in the control group (hazard ratio $1.08,95 \% \mathrm{Cl}$ 0.89-1.31). The incidence of all-cause mortality was also similar in the two groups (hazard ratio 0.93, 95\% Cl 0.67-1.27). Notably, no significant differences were found between the two groups with regard to the incidence of acute myocardial infarction. The authors note, however, that the RECORD trial was never designed to have sufficient power to investigate this issue. On the basis of these results, the investigators conclude that rosiglitazone does not increase the risks of cardiovascular or all-cause mortality in patients with type 2 diabetes.

Original article Home PD et al. (2007) Rosiglitazone evaluated for cardiovascular outcomes-an interim analysis. N Engl J Med 357: 28-38

\section{Chronic kidney disease components associated with risk of cardiovascular disease}

Although chronic kidney disease (CKD) is widely accepted as an independent risk factor for cardiovascular disease (CVD), the associations between individual components of CKD and cardiovascular outcomes have not been characterized. Using data from the National Kidney Foundation's Kidney Early Evaluation Program, McCullough et al. have demonstrated that anemia, estimated glomerular filtration rate (eGFR) and microalbuminuria are all independently associated with risk of CVD.

The study cohort comprised 37,153 individuals (mean age 52.9 years; $68.7 \%$ female) who had attended a National Kidney Foundation screening session designed to identify those at high risk of kidney disease. All participants completed a survey about past medical events. National data systems were used to obtain mortality information.

The prevalence of CVD was approximately fivefold higher in patients with an eGFR of $<30 \mathrm{ml} / \mathrm{min} / 1.73 \mathrm{~m}^{2}$ than in patients with an
eGFR of $\geq 90 \mathrm{ml} / \mathrm{min} / 1.73 \mathrm{~m}^{2}$. An increased prevalence of CVD was also noted in those patients with the highest levels of albuminuria and in those with the lowest levels of hemoglobin. Multivariate analysis adjusted for age identified eight factors that were independently associated with increased risk of CVD: male sex, being a smoker, high BMI, diabetes mellitus, hypertension, eGFR of $30-59 \mathrm{ml} / \mathrm{min} /$ $1.73 \mathrm{~m}^{2}$, hemoglobin level of $\leq 128 \mathrm{~g} / \mathrm{l}$, and urine albumin concentration of $>30 \mathrm{mg} / \mathrm{l}$. Independent predictors of all-cause mortality included male sex, diabetes mellitus, microalbuminuria, prevalent CKD, prevalent CVD, and concurrent CKD and CVD. The authors note that patients with anemia, a low eGFR and microalbuminuria are at particularly high risk of CVD.

Original article McCullough PA et al. (2007) Independent components of chronic kidney disease as a cardiovascular risk state: results from the Kidney Early Evaluation Program (KEEP). Arch Intern Med 167: 1122-1129

\section{Systematic review of techniques for assessing lower limb peripheral arterial disease}

The type of intervention for lower limb peripheral arterial disease is determined by disease severity; therefore, accurate assessment is essential. Intra-arterial contrast angiography is regarded as the reference standard, but it carries risks associated with arterial puncture and ionizing radiation. A UK-based group has performed a systematic review to ascertain whether magnetic resonance (MR) angiography, CT angiography or duplex ultrasonography could be safer and more acceptable to patients than contrast angiography for assessing the severity of this condition.

The investigators identified 107 relevant studies from searches of 11 electronic databases, 6 specialist journals and the reference lists of included papers. Contrast-enhanced MR angiography was the most accurate of the techniques for detection of $50 \%$ or more stenosis (median sensitivity $95 \%$ and median specificity $97 \%$, versus $91 \%$ and $91 \%$ respectively for CT angiography, and $88 \%$ and $96 \%$ respectively for duplex ultrasonography). Although MR angiography was associated with the greatest number of adverse events, these were generally mild; the majority of severe adverse events, although infrequent, were associated with contrast 